\title{
Association between hyperreflective foci in the outer retina, status of photoreceptor layer, and visual acuity in diabetic macular edema.
}

\section{$\operatorname{AUTHOR}(\mathrm{S}):$}

Uji, Akihito; Murakami, Tomoaki; Nishijima, Kazuaki; Akagi, Tadamichi; Horii, Takahiro; Arakawa, Naoko; Muraoka, Yuki; Ellabban, Abdallah A; Yoshimura, Nagahisa

\section{CITATION:}

Uji, Akihito ... [et al]. Association between hyperreflective foci in the outer retina, status of photoreceptor layer, and visual acuity in diabetic macular edema.. American journal of ophthalmology 2012, 153(4): 710-717.e1

\section{ISSUE DATE:}

2012-04

URL:

http://hdl.handle.net/2433/155122

\section{RIGHT:}

(C) 2012 Elsevier Inc.; This is not the published version. Please cite only the published version.; この論文は出版社版でありません。引用の際に は出版社版をご確認ご利用ください。 


\section{ABSTRACT}

PURPOSE: To determine if hyperreflective foci in the outer retina are associated with photoreceptor integrity and the logarithm of minimum angle of resolution (logMAR) visual acuity (VA) in patients with diabetic macular edema (DME).

DESIGN: Retrospective, observational, cross-sectional study.

METHODS: Patients ( $n=76 ; 108$ eyes) with clinically relevant macular edema and no serous retinal detachment were analyzed retrospectively. Spectral-domain optical coherence tomography (SD-OCT) images were obtained for all patients. We investigated the relationship between the hyperreflective foci in the outer retinal layers than the external limiting membrane (ELM) at the fovea and the photoreceptor integrity and VA.

RESULTS: SD-OCT showed that 58 (53.7\%) eyes had hyperreflective foci in the outer retinal layers, and 107 (99.1\%) eyes had hyperreflective foci in the inner retinal layers. The logMAR VA was significantly $(P<.0001)$ worse in eyes with hyperreflective foci in the outer retinal layers than eyes without them $(0.463 \pm 0.382$ vs. $0.127 \pm 0.206$, respectively). Disruption of the ELM line on OCT was significantly 
Hyperreflective foci, photoreceptor layer, and VA in DME. Uji, et al.--2

$(P<.0001$, for both comparisons) associated with both hyperreflective foci in the outer retinal layers and poor logMAR VA. Disruption of the junction of the inner and outer segment line (IS/OS) also was related to hyperreflective foci in the outer retinal layers and poor logMAR VA ( $P<.0001$ for both comparisons).

CONCLUSIONS: The presence of hyperreflective foci in the outer retina is closely associated with a disrupted ELM and IS/OS line on SD-OCT images and decreased VA in DME. 
Association between Hyperreflective Foci in the Outer Retina, Status of Photoreceptor Layer and Visual Acuity in Diabetic Macular Edema

Running title: Hyperreflective foci, photoreceptor layer, and VA in DME

AKIHITO UJI, MD, TOMOAKI MURAKAMI, MD, KAZUAKI NISHIJIMA, MD,

TADAMICHI AKAGI, MD, TAKAHIRO HORII, MD, NAOKO ARAKAWA, MD, YUKI

MURAOKA, MD, ABDALLAH A. ELLABBAN, MD, AND

NAGAHISA YOSHIMURA, MD

From the Department of Ophthalmology and Visual Sciences, Kyoto University

Graduate School of Medicine, Kyoto, Japan.

Corresponding author: Akihito Uji, M.D.

Department of Ophthalmology and Visual Sciences, Kyoto University Graduate

School of Medicine, 54 Shogoin Kawahara-cho, Sakyo-ku, Kyoto 606-8507, Japan;

E-mail: akihito1@kuhp.kyoto-u.ac.jp; Tel: +81-75-751-3248; Fax: +81-75-752-0933. 
Hyperreflective foci, photoreceptor layer, and VA in DME. Uji, et al.--2

\section{INTRODUCTION}

Diabetic macular edema (DME) is a leading cause of visual impairment in patients with diabetes. The Wisconsin Epidemiologic Study of Diabetic Retinopathy reported that the incidence of DME over a 10 -year period was $20.1 \%$ in a younger-onset group, $25.4 \%$ in an older-onset group taking insulin, and $13.9 \%$ in an older-onset group not taking insulin. ${ }^{1}$ Since the number of patients with diabetic retinopathy (DR) and vision-threatening DR is expected to increase ${ }^{2}$ further research is needed into development of treatments and evaluation techniques for DME.

Optical coherence tomography (OCT) has been used widely to assess the pathological structures in the macula and objectively measure the retinal thickness. ${ }^{3-6}$ To date, a modest correlation between OCT-measured central retinal thickness and visual acuity (VA) has been reported to some extent in DME. ${ }^{5-11}$ Despite the clinical relevance of this finding, clinicians frequently have observed eyes with poor visual outcomes regardless of complete resolution of the macular edema, and the VA is highly variable in association with a given degree of retinal edema. ${ }^{12,13}$ These clinical findings suggested that a pathogenesis other than macular thickening may be at play and should be investigated.

In addition to quantification, later generations of OCT also enable clinicians to 
Hyperreflective foci, photoreceptor layer, and VA in DME. Uji, et al.--3

appreciate the individual retinal layers qualitatively. In particular, spectral-domain (SD)-OCT clearly delineates the external limiting membrane (ELM), which corresponds to the adherens junctions between the Müller cells and photoreceptor cells and also prevents passage of macromolecules. ${ }^{14}$ The junction between the inner and outer segments of the photoreceptors (IS/OS) on OCT images might represent the photoreceptor function, which is light perception. These layers on the OCT images reportedly provide important information about pathologies of the photoreceptors. Several studies have reported a correlation between damage to the foveal photoreceptors and the VA in retinal vascular diseases. ${ }^{12,13,15-18}$ Collectively, those studies have suggested both the clinical relevance of the photoreceptor damage and a novel pathogenesis in DME that involves photoreceptor dysfunction and macular thickening.

Recently, Bolz et al. used SD-OCT to show that hyperreflective foci are associated with DME and that these structures are scattered throughout all retinal layers. ${ }^{19}$ The authors postulated that these foci might represent the initial stages in the development of intraretinal hard exudates and that the foci might be subclinical features of lipoprotein extravasation after breakdown of the inner blood-retina 
barrier. Considering this, Ota et al., who have studied hyperreflective dots in serous retinal detachments (SRDs), speculated that fluid and exudates, such as lipids and proteins, that flow into the subretinal space through the outer retinal cleavage may be deposited as subfoveal hard exudates. ${ }^{20-22}$

In the current study, we characterized the sparse hyperreflective foci, subclinical findings that are invisible during clinical ophthalmoscopic examinations, and investigated their association with the photoreceptor status and visual function in DME without SRDs.

\section{PATIENTS AND METHODS}

We retrospectively examined 108 eyes of 76 patients (mean age \pm standard deviation, $65.0 \pm 9.1$ years; range, 35-82 years; 5 patients with type 1 diabetes mellitus [DM], and 71 with type 2 DM) who had visual disturbances due to clinically relevant macular edema and were treated at Kyoto University Hospital between December 2007 and March 2010. All patients had undergone comprehensive ophthalmologic examinations, including measurement of the best-corrected visual acuity (BCVA), slit-lamp biomicroscopy, color fundus photography, and SD-OCT. 
Hyperreflective foci, photoreceptor layer, and VA in DME. Uji, et al.--5

We excluded eyes with a SRD, hard exudates at the fovea, other retinal diseases

(including uveitis, vitreomacular traction syndrome, or macular pucker), or significant media opacities, such as cataract or vitreous hemorrhage.

\section{Optical Coherence Tomography}

The morphologic features of DME, including the ELM, IS/OS, and hyperreflective foci, were assessed with SD-OCT (Spectralis, Heidelberg Engineering, Heidelberg, Germany). Twenty-degree radial scans centered on the fovea were obtained in a clockwise manner in all eyes, and 49 raster scans were used to evaluate the mean retinal thickness (from the innermost internal limiting membrane [ILM] to the retinal pigment epithelium [RPE]) at the fovea (radius, $500 \mu \mathrm{m}$ ). The central fovea was defined as the location without the inner retinal layers in the macular region. ${ }^{23}$ Subsequently, the morphologic features within a scanned 1-mm area centered on the fovea were evaluated for each eye. The status of the ELM and IS/OS was classified according to previously described methods with some modifications. ${ }^{16,18 \text {, }}$ ${ }^{24}$ Briefly, eyes with an ELM line that appeared to be complete at the fovea in all scans, the eyes were classified as ELM (+); eyes with an interrupted ELM line in 
Hyperreflective foci, photoreceptor layer, and VA in DME. Uji, et al.--6

one scan or more were classified as ELM ( \pm ); and eyes with an undetectable ELM line in the fovea were classified as ELM (-). Each eye also was classified based on the status of the IS/OS line beneath the fovea using the same criteria described for the ELM line, i.e., IS/OS (+), IS/OS ( \pm ), and IS/OS (-).

Since a number of studies have reported the photoreceptor status as a novel parameter in macular edema associated with retinal vascular diseases, ${ }^{12,13,15-18,25,}$ ${ }^{26}$ we evaluated the presence of the hyperreflective foci in the outer retinal layers. It was previously reported that ELM acts as a barrier against macromolecules, ${ }^{14}$ which led us to hypothesize that an intact ELM might block diffusion of proteins or lipids from the damaged retinal vasculature, whereas they might otherwise migrate freely within the inner retinal layers. In other words, migration of hyperreflective foci into the outer retinal layers than the ELM might indicate that the barrier properties of the ELM are disrupted or that the inner or outer segments necessary for light perception have been damaged. Thus, we evaluated and defined the hyperreflective foci in the outer retinal layers from the ELM to the RPE within each 1-mm scanned area centered on the fovea (Figure 1). As discussed previously, the ELM was often interrupted or absent at the fovea. When two or more fragments of 
Hyperreflective foci, photoreceptor layer, and VA in DME. Uji, et al.--7

the ELM line were detected in the scanned area, we drew imaginary lines between the ends of the ELM lines (Figure 2). When the ELM line reached the outer side without interruption and ended at the RPE-choriocapillaris complex, we considered the area where the ELM line was absent as being above the ELM line and did not draw an additional line (Figure 2, bottom row). The sparse hyperreflective foci were round or oval as reported previously, ${ }^{19}$ and the hyperreflective foci aggregates were often seen on the OCT images and corresponded to hard exudates in the fundus photographs. We excluded eyes with aggregates within the 1-mm central scanned area (Figure 1), because the aim of this study was to evaluate the association between the photoreceptor status and sparse hyperreflective foci, which are considered as subclinical hard exudates.

Two experienced masked examiners (Y.M., N.A.) classified the status of the ELM and the IS/OS and the presence of the hyperreflective foci, and a third higher level grader (T.H.) classified any images about which there was disagreement. The Kappa coefficient was computed as a measure of reliability between the observers.

\section{Statistical Analysis}


Hyperreflective foci, photoreceptor layer, and VA in DME. Uji, et al.--8

All values are expressed as the mean \pm standard deviation. All BCVA

measurements were converted to the logarithm of the minimum angle of resolution (logMAR) equivalents before statistical analysis. Student's $t$-tests and chi-square tests were used to compare the two hyperreflective foci groups (foci absent in the outer retinal layers group vs. foci present in the outer retinal layers group) regarding gender, age, duration of diabetes, $\mathrm{HbA1C}$, total cholesterol, the presence of hypertension, logMAR VA, type of $\mathrm{DR}$, the hyperreflective foci in the inner retinal layers than ELM line (Figure 1), and the integrity of the IS/OS and the ELM lines. The chi-square test also was used to analyze the relationship between the integrity of the ELM line and the IS/OS line. Comparisons of the logMAR VA levels of the three groups that were classified based on the status of the IS/OS or ELM lines and comparison of the foveal thickness of the three ELM groups were carried out using one-way analysis of variance, with post-hoc comparisons tested by Scheffe procedure. The relationship between the logMAR VA and the foveal thickness was analyzed using the Pearson correlation coefficient. A $P$ value $<.05$ was considered statistically significant. All analyses, except for the kappa coefficient, were performed using StatView version 5.0 (SAS Institute, Cary, NC). Calculation of the 
kappa coefficient was performed using SPSS version 17 (GraphPad Software, La Jolla, CA).

\section{RESULTS}

\section{Assessment of Inter-observer Agreement}

The Kappa coefficient was $0.897(P<.001)$ for the ELM, $0.833(P<.001)$ for the IS/OS, and $0.926(P<.001)$ for location of the hyperreflective foci. These results indicated good inter-observer agreement.

\section{Hyperreflective Foci in the Outer Retinal Layers than the External Limiting}

\section{Membrane}

Recent publications have documented the presence of hyperreflective foci in Spectralis OCT images of DR, but it is unclear whether the finding is related to visual function, which encouraged us to investigate the clinical relevance of hyperreflective foci in DME. We observed hyperreflective foci in the central 1-mm area of the fovea in the inner retinal layers in 107 (99.1\%) of the 108 eyes with DME 
(Figure 1). However, only 58 (53.7\%) of the 108 eyes had hyperreflective foci in the outer retinal layers.

The logMAR VA was significantly $(P<.0001)$ worse in the group with hyperreflective foci in the outer retinal layers compared with the group without hyperreflective foci in the outer retinal layers (Table 1), although no other systemic or ocular parameters differed between the groups. We further investigated how hyperreflective foci in the outer retinal layers contributed to the pathogenesis of DME and found that 45 of 50 eyes (90\%) without hyperreflective foci in the outer retinal layers had an intact ELM, whereas $45(77.6 \%)$ of 58 eyes with hyperreflective foci in the outer retinal layers had a disrupted $\operatorname{ELM}(P<.0001)$ (Table 2). The logMAR VA in the ELM (+) group also was significantly better than that in either the ELM $( \pm)$ or ELM $(-)$ groups $(P<.0001$ for both comparisons).

These results suggested that the pathological association between the hyperreflective foci in the outer retinal layers and disrupted ELM may contribute to visual dysfunction in DME.

\section{Association between Hyperreflective Foci and Photoreceptor Status}


Despite the clinical relevance of ELM disruption in the OCT images, which affects visual function but not light perception or signal transduction per se ${ }^{14}$ the major function of the ELM seems to be that of a barrier. We also evaluated another marker of photoreceptor integrity, the IS/OS, which directly represents light perception. We found that eyes with an intact IS/OS had significantly better VA than eyes with either an interrupted or absent IS/OS ( $P=.0021$ and $P<.0001$, respectively). All 27 eyes with an intact IS/OS also had an intact ELM, whereas 50 (61.7\%) of 81 eyes with a disrupted IS/OS had either an interrupted or absent ELM $(P<.0001)$ (Table 3). In addition, $96.3 \%$ of eyes with an intact IS/OS had no hyperreflective foci in the outer retinal layers, especially compared to the higher frequency of hyperreflective foci in the outer retinal layers $(71.3 \%)$ in eyes with a disrupted IS/OS $(P<.0001)($ Table 3$)$. These results suggested that the pathological association between the hyperreflective foci in the outer retinal layers and a disrupted ELM may affect the damage in the cone inner or outer segments and the visual function.

\section{Modest Association between Foveal Thickness and Hyperreflective Foci}

The macular thickness might represent the magnitude of the macular edema, the 
Hyperreflective foci, photoreceptor layer, and VA in DME. Uji, et al.--12

severity of the damage in signal transduction, or both. Thus, when we investigated whether the hyperreflective foci in the outer retinal layers were associated with the macular thickening, we found that the mean foveal thickness was significantly $(P$ $=.0081$ ) higher in the group with hyperreflective foci in the outer retinal layers than in the group with no hyperreflective foci in the outer retinal layers (Table 4). In addition, the mean foveal thickness was correlated significantly $(P=.0033)$ with the ELM status at the fovea (Table 4) and the mean foveal thickness was modestly correlated with the logMAR VA $(R=0.275 ; P=.0038)$.

\section{DISCUSSION}

A number of studies have proposed that disruption of the photoreceptors in eyes with macular edema results from retinal vascular disease as a novel parameter that predicts visual function. ${ }^{12,13,15-18,25,26}$ However, it is unclear what induces the photoreceptor damage after the breakdown of the blood-retinal barrier. The current study showed for the first time that hyperreflective foci in the outer retinal layers are associated with the disrupted photoreceptor markers, ELM and IS/OS, and concomitant visual impairment, which suggested both novel pathological 
Hyperreflective foci, photoreceptor layer, and VA in DME. Uji, et al.--13

mechanisms and their clinical relevance. However, the limitations of this study were its retrospective nature and small sample size.

Bolz et al. previously suggested that hyperreflective foci may represent subclinical features of lipoprotein extravasation that act as precursors of hard exudates. ${ }^{19}$ Those authors pointed out that well-demarcated hyperreflective foci were seen on Spectralis OCT scans in patients with DME and that these foci were distributed throughout all retinal layers. Our current study showed that hyperreflective foci were present in the fovea in most eyes with DME, whereas only 58 eyes $(53.7 \%)$ had hyperreflective foci in the outer retinal layers than the ELM. As mentioned previously, the ELM corresponds to the adherens junctions between the Müller cells and photoreceptor cells and acts as a barrier against macromolecules. Thus, we hypothesized that the physiologic ELM restricts migration of lipoproteins or proteins extravasated from the retinal vasculature, which is in the inner retinal layers. Disruption of the ELM might permit these macromolecules to pass through this barrier and be deposited into the outer retinal layers. Another possible explanation is that hyperreflective foci result from a neurodegenerative process;

Baumüller et al. previously documented outer retinal hyperreflective spots in 
Hyperreflective foci, photoreceptor layer, and VA in DME. Uji, et al.--14

macular telangiectasia type $2 .{ }^{27}$ In the current study, we found that the ELM lines merged with the RPE in about half of the eyes in the ELM ( \pm ) group (Figure 2, bottom row). In those cases, the absence of the ELM and IS/OS lines in those areas might represent cellular damage or death, whereas the hyperreflective foci and irregular RPE lines detected at a high frequency in those areas may represent degenerated retinal cells themselves or macrophages attempting to engulf the cells. ${ }^{27,28}$

Increasing amounts of hard exudates are associated with a risk of visual impairment in patients with DR, and deposition of submacular hard exudates commonly causes a severe decrease in VA. ${ }^{20,29,30}$ Intriguingly, Ota et al. reported that hyperreflective dots in the subretinal spaces were significantly associated with deposition of subfoveal hard exudates after resolution of a SRD. ${ }^{20}$ Assuming that hyperreflective foci represent subclinical lipid extravasation as a precursor of hard exudates and can permeate into the area under the ELM line, the hyperreflective foci themselves are thought to disrupt the foveal photoreceptor layer and the photoreceptor degeneration may already have begun by the time this process was under way, when hard exudates are clinically invisible. 
The significant association between hyperreflective foci in the outer retinal layers and a disrupted ELM suggests several possibilities: a cause-result relationship, a temporal sequence, and an epiphenomenon. Considering that the ELM corresponds to the adherens junctions between the Müller cells and photoreceptors, disruption of the ELM on OCT images might represent degeneration of the Müller cells, photoreceptors, or both, or alternatively, disappearance or disorganization of the heterophilic adherens junctions.

As discussed previously, the breakdown of the barrier properties of the ELM might permit macromolecules or lipid-laden macrophages to migrate into the outer retinal layers, which agrees with the results of a previous report that documented outer retinal discontinuity in eyes with retinal vein occlusion. ${ }^{31}$ Further, extravasated lipoproteins or proteins might contribute to degeneration of the photoreceptor cells or decreased junctional protein expression. Hyperreflective foci in the outer retinal layers beneath an intact ELM especially might represent degenerated photoreceptor cells and ultimately result in a fragmentation of the ELM. A future longitudinal study should elucidate the detailed processes in this pathological interaction. 
Damage to the IS/OS line and the ELM also was correlated with the presence of hyperreflective foci in the outer retinal layers. The outer segments of the photoreceptor cells are the major components of light perception, and their metabolism is regulated in the inner segments. Thus, structural disturbance of the IS/OS lines on the OCT images might represent more directly the functionally impaired light perception, which might correspond well with the fragmented IS/OS lines on OCT images. Further, the extravasated macromolecules might lead to both photoreceptor disorganization and deposition of hyperreflective foci. These pathogeneses might begin after the ELM is disrupted, which agrees with the significant association between the status of the ELM and IS/OS lines. However, the appearance of the IS/OS lines differed in several eyes, i.e., merging of the ELM or IS/OS lines with the RPE, and represented the absence of photoreceptor cells in that area (Figure 2, bottom row). Hyperreflective foci might correspond to degenerated photoreceptors or the macrophages engulfing them. Irregular RPE lines were sometimes seen on the OCT images in the current study and may indicate macrophages on the RPE line, activated or overphagocytosed RPE cells, or RPE metaplasia. ${ }^{22,30,32}$ Considering that there was a higher frequency of 
interrupted or absent IS/OS lines than of similar ELM lines, the pathological interaction between degenerated photoreceptors and RPE cells might result in the precedence of disrupted IS/OS (Figures 2, 3).

Macular thickness is a well-accepted parameter associated with visual disturbance in DME. ${ }^{5-11}$ In the current study, eyes with hyperreflective foci in the outer retinal layers were thicker than those without hyperreflective foci in the outer retinal layers. Thus, it is reasonable that severe breakdown of the blood-retinal barrier might result in thickening of the retinal parenchyma and extravasation of macromolecules or macrophages. Interestingly, the ELM status also was associated with the macular thickness, which led us to speculate further that degeneration or dysfunction of the Müller cells may cause the ELM lines to disappear, leading to structural fragility and a concomitant increase in the volume of the retinal parenchyma.

The current study showed the clinical relevance of the hyperreflective foci, which are otherwise clinically invisible, in the outer retinal layers on the Spectralis OCT images. We also identified a pathological association between hyperreflective foci in the outer retinal layers and disrupted ELM or IS/OS lines, suggesting 
Hyperreflective foci, photoreceptor layer, and VA in DME. Uji, et al.--18

photoreceptor degeneration in DME. 


\section{ACKNOWLEDGMENTS}

A. Funding/Support: none.

B. Financial Disclosures: none.

C. Contributions of Authors: Contributions of Authors: design of the study (A.U.,

T.M., K.N., N.Y.); conduct of the study (A.U., T.M., K.N., T.A., T.H., N.A., Y.M., A.A.E., N.Y.); collection of data (A.U., T.M., K.N., T.A., T.H., N.A., Y.M., A.A.E., N.Y.); interpretation of data (A.U., T.M., K.N., N.Y.); statistical analysis of data (A.U., T.M., K.N., N.Y.); and preparation, review, or approval of the manuscript (A.U, T.M., K.N, T.A., T.H., N.A., Y.M., A.A.E., N.Y.).

D. Ethics Committee Approval: All the research and measurements adhered to the tenets of the Declaration of Helsinki and were approved by the institutional review board at Kyoto University Graduate School of Medicine. Each patient provided informed consent after a detailed explanation of the nature and possible consequences of the study procedures.

E. Other Acknowledgments: none. 


\section{REFERENCES}

1. Klein R, Klein BE, Moss SE, Cruickshanks KJ. The Wisconsin Epidemiologic Study of Diabetic Retinopathy. XV. The long-term incidence of macular edema. Ophthalmology 1995;102(1):7-16.

2. Saaddine JB, Honeycutt AA, Narayan KM, Zhang X, Klein R, Boyle JP. Projection of diabetic retinopathy and other major eye diseases among people with diabetes mellitus: United States, 2005-2050. Arch Ophthalmol 2008;126(12):1740-1747.

3. Otani T, Kishi S, Maruyama Y. Patterns of diabetic macular edema with optical coherence tomography. Am J Ophthalmol 1999;127(6):688-693.

4. Otani T, Kishi S. Tomographic findings of foveal hard exudates in diabetic macular edema. Am J Ophthalmol 2001;131(1):50-54.

5. Hee MR, Puliafito CA, Wong C, et al. Quantitative assessment of macular edema with optical coherence tomography. Arch Ophthalmol 1995;113(8):1019-1029.

6. Browning DJ, Glassman AR, Aiello LP, et al. Relationship between optical coherence tomography-measured central retinal thickness and visual acuity in diabetic macular edema. Ophthalmology 2007;114(3):525-536.

7. Bandello F, Polito A, Del Borrello M, Zemella N, Isola M. "Light" versus "classic" laser treatment for clinically significant diabetic macular oedema. $\mathrm{Br} \mathrm{J}$ Ophthalmol 2005;89(7):864-870.

8. Martidis A, Duker JS, Greenberg PB, et al. Intravitreal triamcinolone for refractory diabetic macular edema. Ophthalmology 2002;109(5):920-927.

9. Laursen ML, Moeller F, Sander B, Sjoelie AK. Subthreshold micropulse diode laser treatment in diabetic macular oedema. $\mathrm{Br} \mathrm{J}$ Ophthalmol 2004;88(9):1173-1179.

10. Ozdemir H, Karacorlu M, Karacorlu SA. Regression of serous macular detachment after intravitreal triamcinolone acetonide in patients with diabetic macular edema. Am J Ophthalmol 2005;140(2):251-255.

11. Massin P, Duguid G, Erginay A, Haouchine B, Gaudric A. Optical coherence tomography for evaluating diabetic macular edema before and after vitrectomy. Am J Ophthalmol 2003;135(2):169-177.

12. Alasil T, Keane PA, Updike JF, et al. Relationship between optical 
coherence tomography retinal parameters and visual acuity in diabetic macular edema. Ophthalmology 2010;117(12):2379-2386.

13. Maheshwary AS, Oster SF, Yuson RM, Cheng L, Mojana F, Freeman WR. The association between percent disruption of the photoreceptor inner segment-outer segment junction and visual acuity in diabetic macular edema. Am J Ophthalmol 2010;150(1):63-67 e61.

14. Marmor MF. Mechanisms of fluid accumulation in retinal edema. Doc Ophthalmol 1999;97(3-4):239-249.

15. Forooghian F, Stetson PF, Meyer SA, et al. Relationship between photoreceptor outer segment length and visual acuity in diabetic macular edema. Retina 2010;30(1):63-70.

16. Sakamoto A, Nishijima K, Kita M, Oh H, Tsujikawa A, Yoshimura N. Association between foveal photoreceptor status and visual acuity after resolution of diabetic macular edema by pars plana vitrectomy. Graefes Arch Clin Exp Ophthalmol 2009;247(10):1325-1330.

17. Otani T, Yamaguchi Y, Kishi S. Correlation between visual acuity and foveal microstructural changes in diabetic macular edema. Retina 2010;30(5):774-780.

18. Kameda T, Nishijima K, Unoki N, et al. Geographic pattern of central retinal sensitivity after intravitreal triamcinolone for diabetic macular edema. Graefes Arch Clin Exp Ophthalmol 2011;249(1):3-9.

19. Bolz M, Schmidt-Erfurth U, Deak G, Mylonas G, Kriechbaum K, Scholda C. Optical coherence tomographic hyperreflective foci: a morphologic sign of lipid extravasation in diabetic macular edema. Ophthalmology 2009;116(5):914-920. 20. Ota M, Nishijima K, Sakamoto A, et al. Optical coherence tomographic evaluation of foveal hard exudates in patients with diabetic maculopathy accompanying macular detachment. Ophthalmology 2010;117(10):1996-2002. 21. Fong DS, Segal PP, Myers F, Ferris FL, Hubbard LD, Davis MD. Subretinal fibrosis in diabetic macular edema. ETDRS report 23. Early Treatment Diabetic Retinopathy Study Research Group. Arch Ophthalmol 1997;115(7):873-877. 22. Sigurdsson R, Begg IS. Organised macular plaques in exudative diabetic maculopathy. Br J Ophthalmol 1980;64(6):392-397.

23. Murakami T, Nishijima K, Sakamoto A, Ota M, Horii T, Yoshimura N. Foveal cystoid spaces are associated with enlarged foveal avascular zone and microaneurysms in diabetic macular edema. Ophthalmology 2011;118(2):359-367. 
24. Yamaike N, Tsujikawa A, Ota M, et al. Three-dimensional imaging of cystoid macular edema in retinal vein occlusion. Ophthalmology 2008;115(2):355-362 e352.

25. Ota M, Tsujikawa A, Murakami T, et al. Foveal photoreceptor layer in eyes with persistent cystoid macular edema associated with branch retinal vein occlusion. Am J Ophthalmol 2008;145(2):273-280.

26. Murakami T, Tsujikawa A, Ohta M, et al. Photoreceptor status after resolved macular edema in branch retinal vein occlusion treated with tissue plasminogen activator. Am J Ophthalmol 2007;143(1):171-173.

27. Baumuller S, Charbel Issa P, Scholl HP, Schmitz-Valckenberg S, Holz FG. Outer retinal hyperreflective spots on spectral-domain optical coherence tomography in macular telangiectasia type 2. Ophthalmology 2010;117(11):2162-2168.

28. Schuman SG, Koreishi AF, Farsiu S, Jung SH, Izatt JA, Toth CA. Photoreceptor layer thinning over drusen in eyes with age-related macular degeneration imaged in vivo with spectral-domain optical coherence tomography. Ophthalmology 2009;116(3):488-496 e482.

29. Takaya K, Suzuki Y, Mizutani H, Sakuraba T, Nakazawa M. Long-term results of vitrectomy for removal of submacular hard exudates in patients with diabetic maculopathy. Retina 2004;24(1):23-29.

30. Takagi H, Otani A, Kiryu J, Ogura Y. New surgical approach for removing massive foveal hard exudates in diabetic macular edema. Ophthalmology 1999;106(2):249-256; discussion 256-247.

31. Tsujikawa A, Sakamoto A, Ota M, et al. Serous retinal detachment associated with retinal vein occlusion. Am J Ophthalmol 2010;149(2):291-301 e295. 32. Murata T, Ishibashi T, Inomata H. Immunohistochemical detection of extravasated fibrinogen (fibrin) in human diabetic retina. Graefes Arch Clin Exp Ophthalmol 1992;230(5):428-431. 


\section{FIGURE CAPTIONS}

\section{FIGURE 1. Localization of hyperreflective foci at the fovea.}

Confluent hyperreflective foci (black arrow) on optical coherence tomography (OCT) images in diabetic retinopathy (top right), which correspond to subfoveal hard exudates in a color fundus photograph (top left). OCT shows sparse hyperreflective foci (black arrows) in the inner retinal layers than the external limiting membrane (middle right) or the outer retinal layers (bottom right) at the fovea, whereas hard exudates are not seen in the corresponding area in the color fundus photographs (middle and bottom left).

FIGURE 2. A disrupted external limiting membrane (ELM) and the junction between the inner and outer segments in eyes with hyperreflective foci in the outer retinal layers in diabetic retinopathy.

Eyes with hyperreflective foci in the outer retinal layers have an intact (top row), discontinuous (second row), or absent (third row) ELM on the optical coherence tomography images. (Top row) The ELM line is intact and the hyperreflective foci are present beneath the line (black arrow), whereas discontinuity of the 
photoreceptor inner segment/outer segment (IS/OS) line (black arrowhead) is seen. (Second and third rows) The ELM line is not continuous in the area between the black arrows. Imaginary lines (red dotted lines) connect the terminals of the ELM and hyperreflective foci are observed beneath the lines (black arrowheads). (Second row) The fragments of both the ELM line (black arrows) and the IS/OS line (asterisk) are depicted within a 1-mm central scanned area. (Third row) The ELM and IS/OS lines are absent. (Bottom row) The ELM lines sometimes sank and merged with the retinal pigment epithelium (RPE) (black arrows). An irregular RPE line (red dotted line) and hyperreflective foci (black arrowheads) are seen in the areas where the ELM and IS/OS are absent.

\section{FIGURE 3. The integrity of the external limiting membrane (ELM) in eyes} without hyperreflective foci in the outer retinal layers.

(Top) In eyes without hyperreflective foci in the outer retinal layers, the ELM (black arrow) and the photoreceptor inner segment/outer segment (IS/OS) (black arrowhead) are frequently intact. (Bottom) A discrepancy between the ELM (black arrow) and the IS/OS (black arrowhead) status is sometimes seen on the optical 
Hyperreflective foci, photoreceptor layer, and VA in DME. Uji, et al.--25

coherence tomography images. 


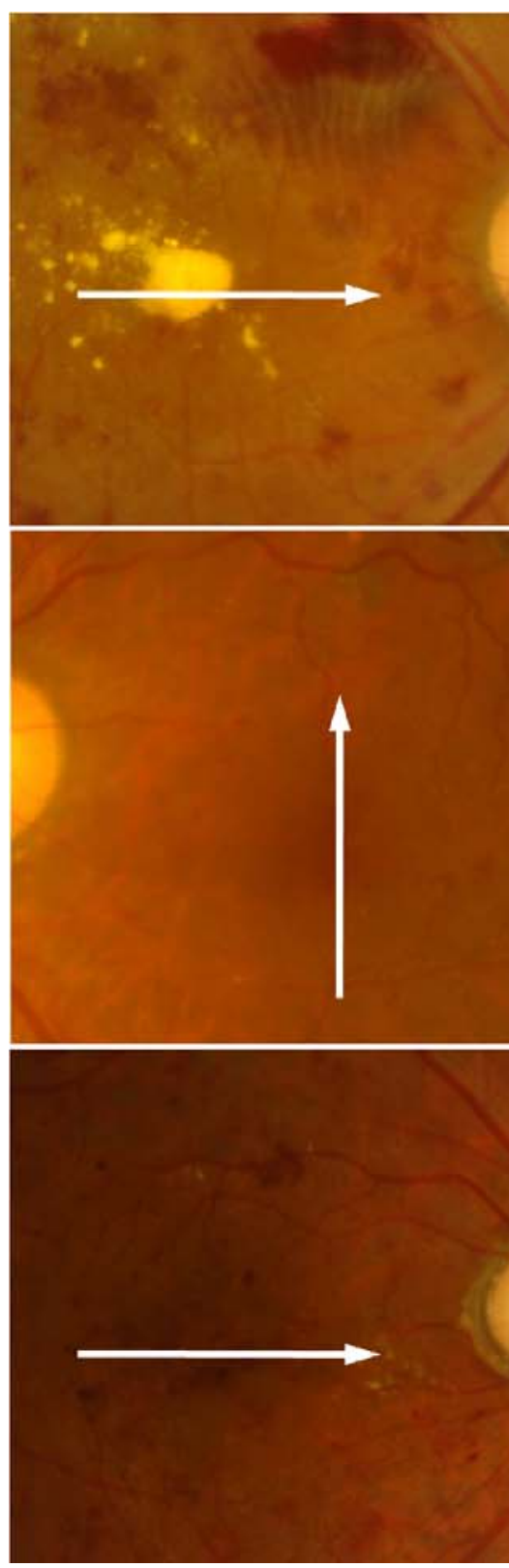

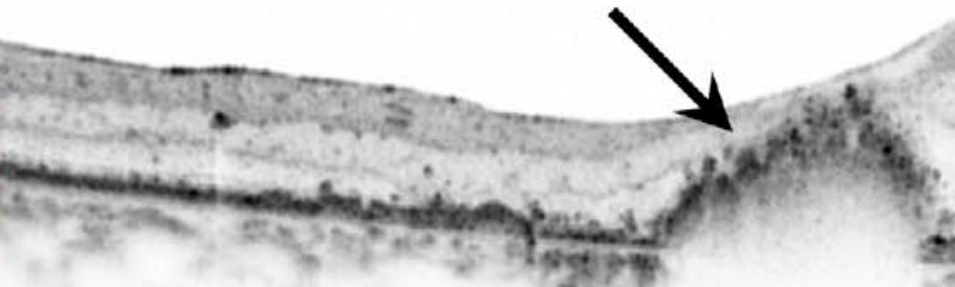

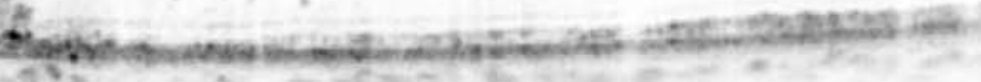
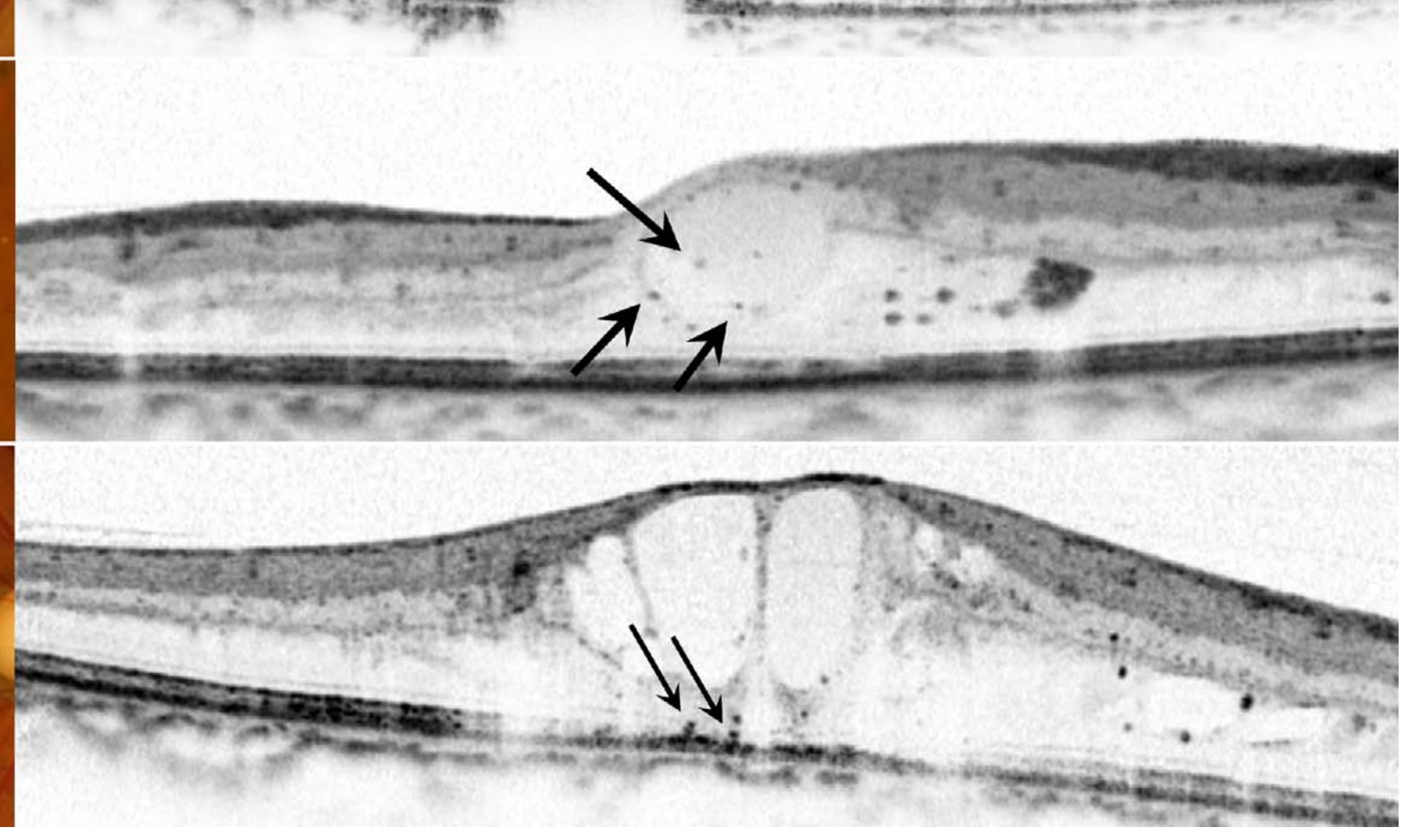

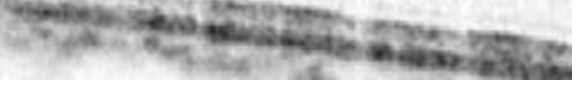



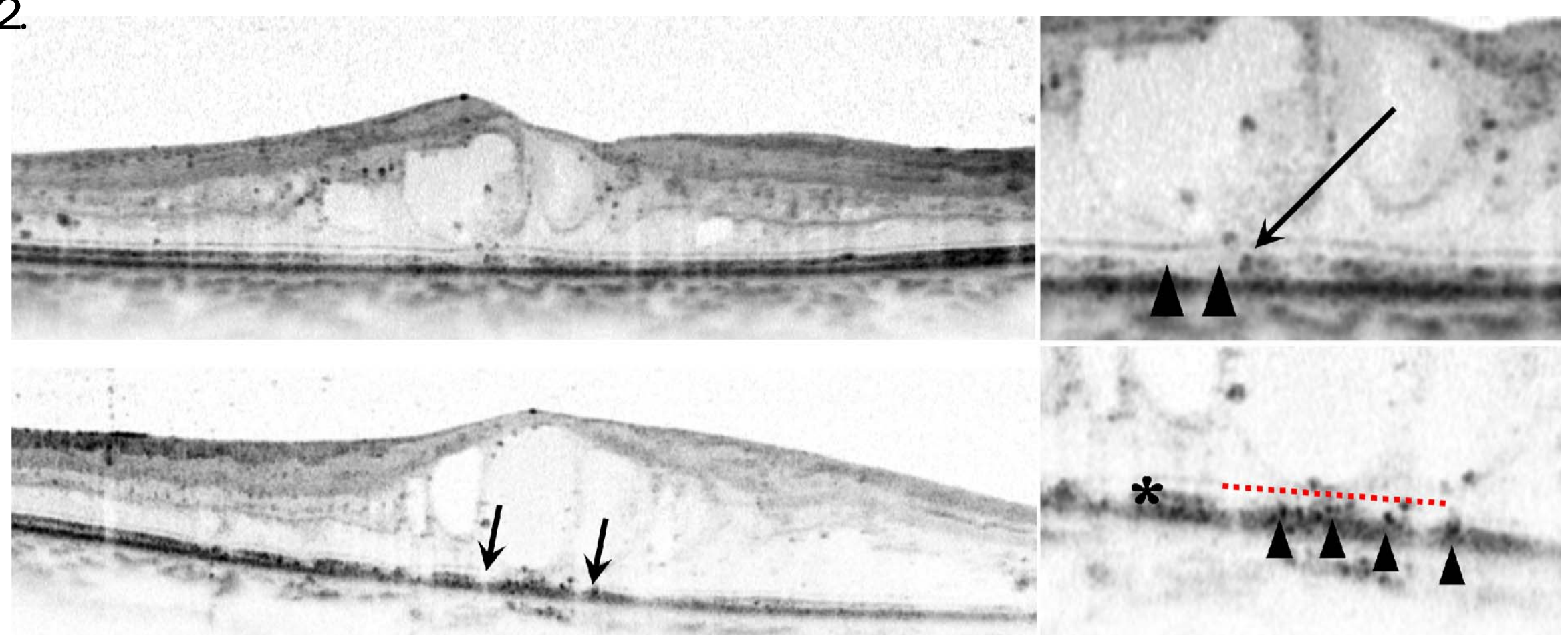

4tosition
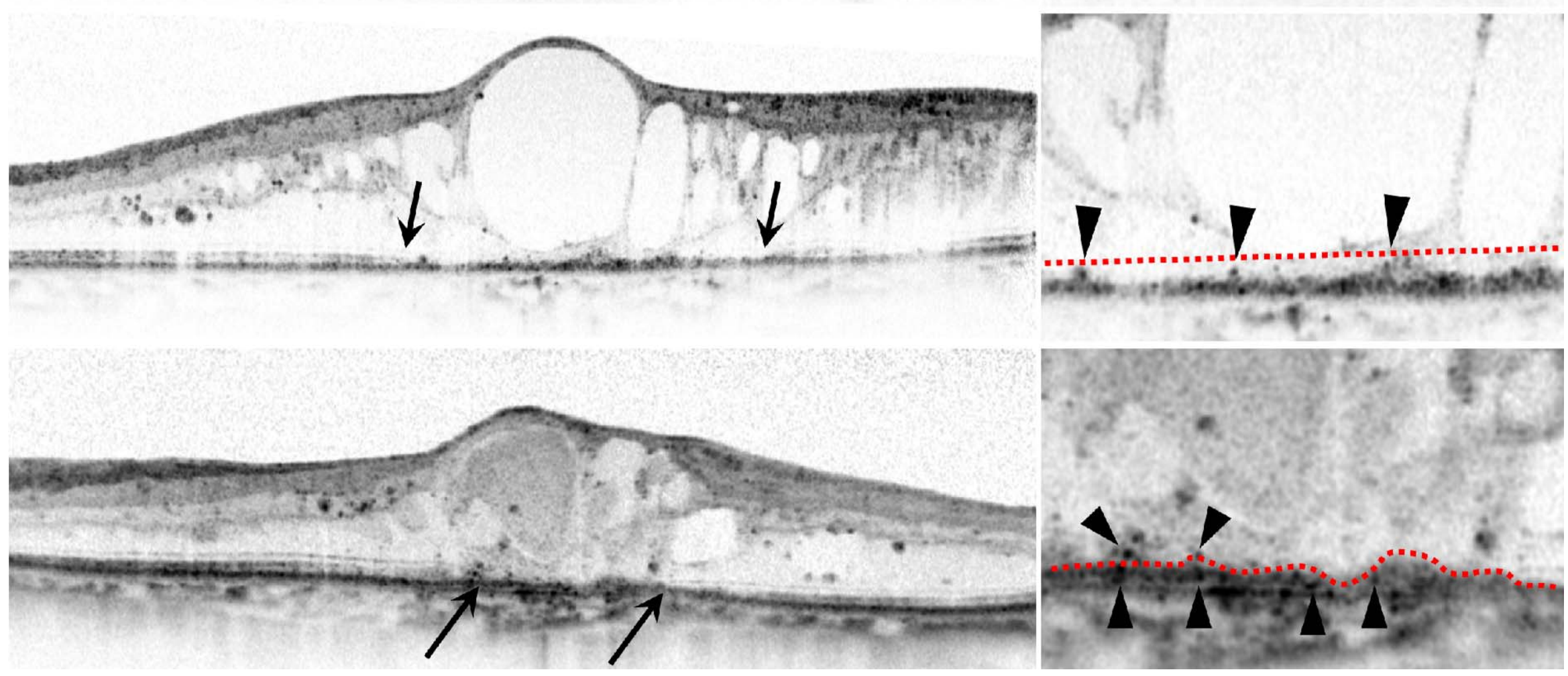
FIGURE 3.
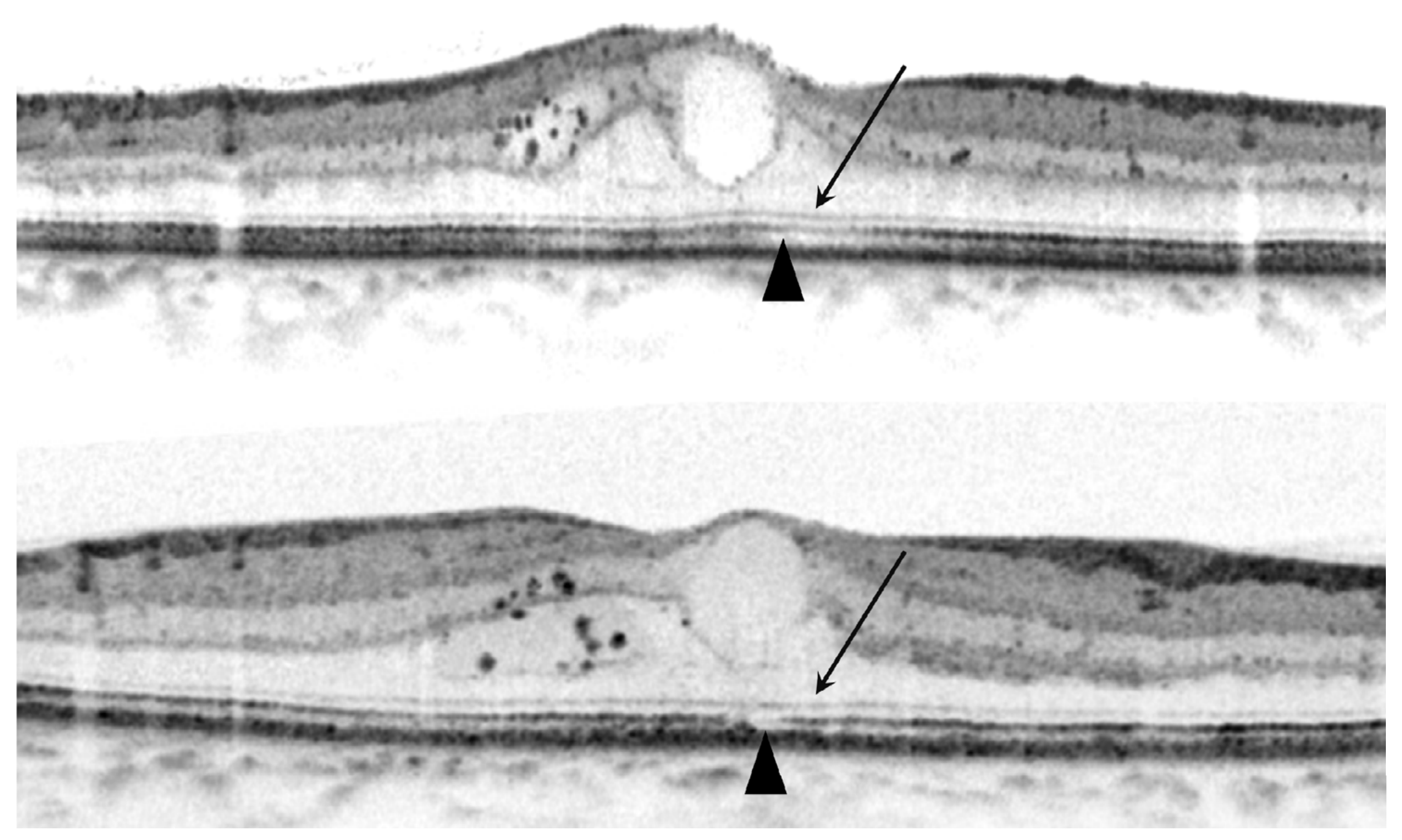

4.

(3)
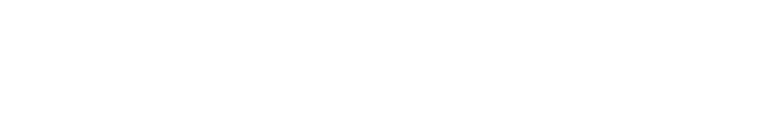

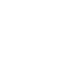




\section{Table of Contents}

(AJO-11-312)

" Association between Hyperreflective Foci in the Outer Retina, Status of Photoreceptor Layer and Visual Acuity in Diabetic Macular Edema."

This retrospective study evaluated the association between the presence of hyperreflective foci in the outer retina and the status of photoreceptor layer and the visual acuity in diabetic macular edema using spectral-domain optical coherence tomography. Hyperreflective foci beneath the external limiting membrane line correlated well with a disrupted external limiting membrane line and junction of the inner and outer segments line and were associated with visual impairment. 
TABLE 1. Differences in Visual Acuity between Eyes with or without Hyperreflective Foci in the Outer Retinal Layers than the External Limiting Membrane

\begin{tabular}{lccc}
\hline Characteristic & $\begin{array}{c}\text { Eyes with Hyperreflective Foci } \\
\text { in Outer Retinal Layers }\end{array}$ & $\begin{array}{c}\text { Eyes without Hyperreflective Foci } \\
\text { in Outer Retinal Layers }\end{array}$ & $P$ Value \\
\hline No. eyes/patients & $58 / 35$ & $50 / 41$ & - \\
Men/women & $28 / 30$ & $27 / 23$ & .5530 \\
Age (years) & $65.7 \pm 8.2$ & $64.3 \pm 10.1$ & .1330 \\
Diabetes duration (years) & $16.9 \pm 8.8$ & $17.6 \pm 9.7$ & .0686 \\
HbA1C (\%) & $7.22 \pm 1.39$ & $7.26 \pm 1.09$ & .8892 \\
Total cholesterol (mg/dl) & $213 \pm 44$ & $198 \pm 35$ & .1377 \\
Hypertension, no. (\%) & $28(48.3)$ & $24(48.0)$ & .9772 \\
Visual acuity (LogMAR) & $0.463 \pm 0.382$ & $0.127 \pm 0.206$ & $<.0001$ \\
DR type, no. (\%) & $0(0.0)$ & $1(2.0)$ & \\
$\quad$ & $19(32.8)$ & $18(36.0)$ & \\
$\quad$ Mild NPDR & $20(34.5)$ & $18(36.0)$ & .6428 \\
$\quad$ Severe NPDR & $19(32.8)$ & $13(26.0)$ & .2792 \\
$\quad$ PDR & $58(100.0)$ & $49(98.0)$ & \\
No. eyes with hyperreflective & & & \\
foci in inner retinal layers (\%) & LogMAR = logarithm of minimum angle of resolution; DR = diabetic retinopathy; NPDR = nonproliferative diabetic \\
\hline Lotinopathy; PDR = proliferative diabetic retinopathy. & & \\
\hline
\end{tabular}


TABLE 2. Association between Hyperreflective Foci in the Outer Retinal Layers and the Status of the External Limiting Membrane Seen on Spectral-Domain Optical Coherence Tomography

\begin{tabular}{lcccc}
\hline & ELM $(+)$ & ELM $( \pm)$ & ELM $(-)$ & $P$ Value \\
\hline Hyperreflective foci in & & & & \\
outer retinal layers & & & & \\
$\quad$ Absent (no. eyes) & 45 & 5 & 0 & \\
$\quad$ Present (no. eyes) & 13 & 39 & 6 & $<.0001$ \\
Visual acuity (logMAR) & $0.153 \pm$ & $0.416 \pm 0.370$ & $1.008 \pm 0.229$ & $<.0001$ \\
& 0.207 & & & \\
\hline ELM = external limiting membrane; logMAR = logarithm of the minimum angle of \\
resolution.
\end{tabular}


TABLE 3. Damaged Junction between the Inner and Outer Segments of the Photoreceptors in Eyes with a Disrupted External Limiting Membrane and Hyperreflective Foci in the Outer Retinal Layers

\begin{tabular}{lcccc}
\hline & IS/OS (+) & IS/OS $( \pm)$ & IS/OS (-) & $P$ Value \\
\hline Visual acuity (logMAR) & $0.060 \pm 0.182$ & $0.299 \pm 0.294$ & $0.733 \pm 0.385$ & $<.0001$ \\
\hline ELM (+) & 27 & 31 & 0 & \\
ELM ( \pm ) & 0 & 33 & 11 & \\
ELM (-) & 0 & 0 & 6 & $<.0001$ \\
\hline Hyperreflective foci in & & & & \\
outer retinal layers & & & 1 & \\
$\quad$ Absent (no. eyes) & 26 & 41 & 16 & $<.0001$ \\
$\quad$ Present (no. eyes) & 1 & & & \\
\hline IS/OS = the junction between the inner and outer segments of the photoreceptors; \\
ELM = external limiting membrane; logMAR = logarithm of minimum angle of \\
resolution.
\end{tabular}


TABLE 4. Correlation between Foveal Thickness and the External Limiting Membrane and Hyperreflective Foci in the Outer Retina

\begin{tabular}{lcc}
\hline & Foveal Thickness $(\mu \mathrm{m})$ & $P$ Value \\
\hline Visual acuity (logMAR) & $404 \pm 115$ & $.0038 ; R=0.275$ \\
\hline ELM (+) & $370 \pm 74$ & \\
ELM $( \pm)$ & $441 \pm 132$ & .0033 \\
ELM (-) & $461 \pm 207$ & \\
\hline Hyperreflective foci in & \\
outer retinal layers & \\
$\quad$ Absent & $373 \pm 78$ & .0081 \\
$\quad$ Present & $431 \pm 135$ & \\
ELM = external limiting membrane; logMAR = logarithm of minimum angle \\
of resolution.
\end{tabular}




\section{BIOSKETCH}

Akihito Uji, M.D., Ph.D. is an Assistant Professor of Ophthalmology at Kyoto

University, Kyoto, Japan. He received his MD and PhD degrees from Okayama

University Graduate School of Medicine, Okayama, Japan. His interests are diabetic retinopathy and image analysis studies. 


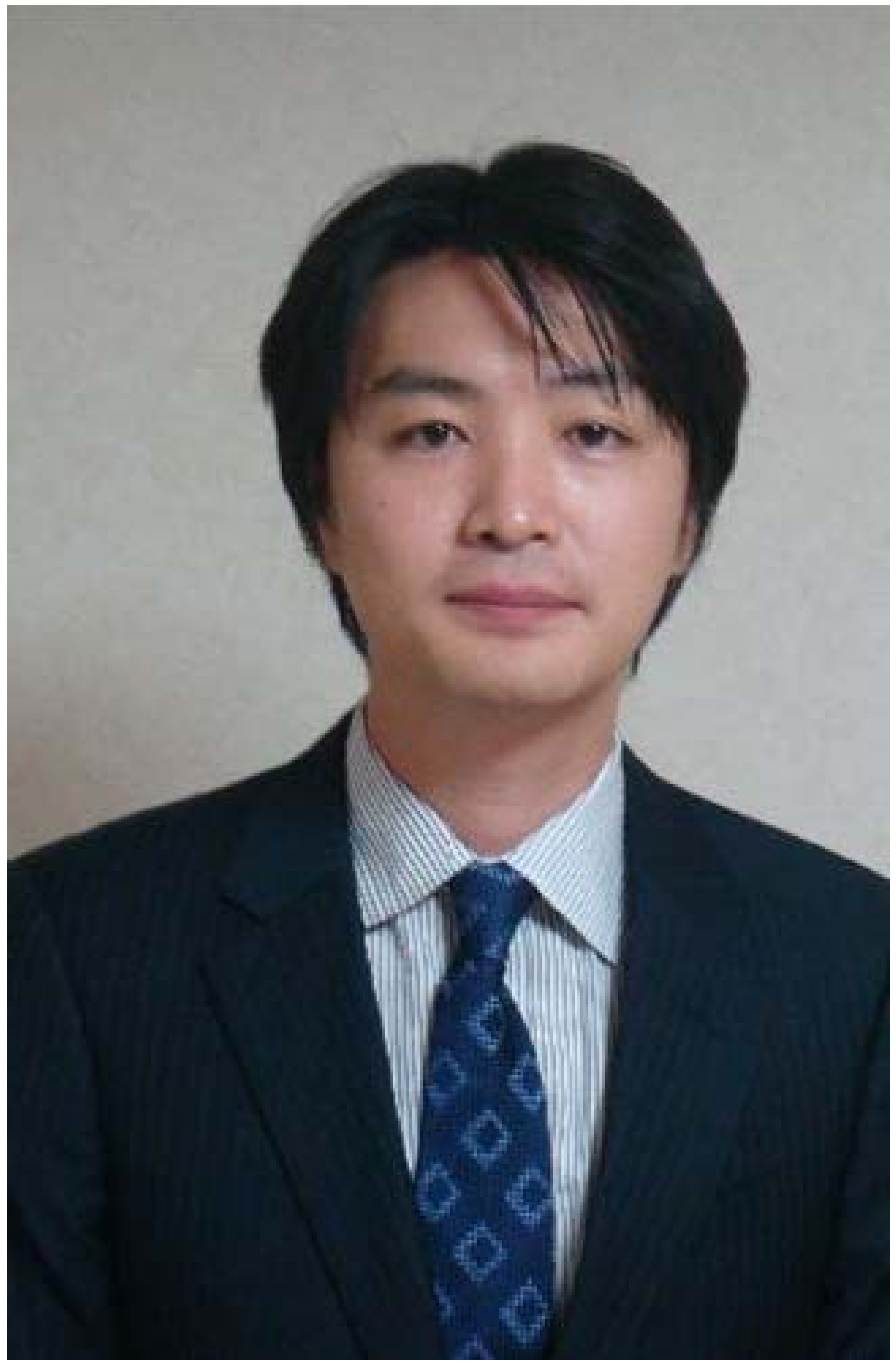

